\title{
Magnetic hysteresis of interface-biased flat iron dots
}

\author{
G. O. G. Rebouças, ${ }^{1}$ A. S. W. T. Silva, ${ }^{1}$ Ana L. Dantas, ${ }^{2}$ R. E. Camley,${ }^{3}$ and A. S. Carriço ${ }^{1}$ \\ ${ }^{1}$ Departamento de Física, Universidade Federal do Rio Grande do Norte, 59072-970-Natal-RN, Brazil \\ ${ }^{2}$ Departamento de Física, Universidade do Estado do Rio Grande do Norte, 59610-210-Mossoró-RN, Brazil \\ ${ }^{3}$ Department of Physics, University of Colorado at Colorado Springs, Colorado Springs, Colorado 80918, USA
}

(Received 22 July 2008; published 4 March 2009)

\begin{abstract}
We report a theoretical study of the coercivity and bias of iron dots exchange coupled with an antiferromagnetic substrate. We show that flat dots, with height close to the iron exchange length, and lateral dimensions of a few exchange lengths, exhibit large enhancement of coercivity and exchange bias. For small interface field strength the magnetization reversal is nearly a coherent rotation with symmetrical loops. Interface pinning leads to large reduction in coercivity and asymmetrical loops, if the interface field strength is comparable to the value of the iron exchange field. We discuss the impact of geometrical confinement and interface pinning on the magnetization reversal mechanisms. We show that small area dots with height larger than the exchange length display stronger interface effects.
\end{abstract}

DOI: 10.1103/PhysRevB.79.104402

PACS number(s): 75.75.+a, 75.70.Ak

\section{INTRODUCTION}

The motivation for studies of arrays of magnetic particles stems from both fundamental reasons and potential technological applications. ${ }^{1-8}$ Sub-100 nm soft particles have dimensions comparable to the exchange length, and the magnetic states and reversal mechanisms depend on their geometrical shapes and sizes. ${ }^{9-15}$

Patterned media, with data storage at one particle per bit basis are currently considered as a promising way of achieving higher storage densities for longitudinal recording. ${ }^{5-7}$ The remanence and coercivity are key parameters for these applications, and control the stray field and the thermal stability. Both parameters can be tailored by exchange coupling the ferromagnetic $(\mathrm{F})$ nanoelement to an extended antiferromagnetic (AF) substrate. These issues have recently been investigated in experimental studies of arrays of interface biased ferromagnetic dots, $\mathrm{Fe} / \mathrm{FeF}_{2},{ }^{16-19} \mathrm{Ni} / \mathrm{NiO},{ }^{21}$ $\mathrm{NiFe} / \mathrm{CoO},{ }^{22,23} \mathrm{NiFe} / \mathrm{IrMn},{ }^{24}$ and $\mathrm{Co} / \mathrm{CoO} .{ }^{25}$

Early reports have shown that the exchange bias of $\mathrm{Fe} / \mathrm{FeF}_{2}$ patterned bilayers can be tuned by their lateral dimensions. ${ }^{16}$ Nanostructuring of nanoporous $\mathrm{FeF}_{2} / \mathrm{Fe}$ bilayers produces major changes in the magnetostatic energy, leading to pronounced asymmetry of the magnetic energy, large exchange bias, and reduced coercivity. ${ }^{19}$

Knowledge of the reversal mechanisms of interface biased dots might help understanding key features of F/AF bilayers with extended interfaces. The study of exchange bias, the shift of hysteresis loops of ferromagnetic films exchange coupled to AF substrates, might benefit from the small interface area of nanometric dots, which opens a chance of probing defect-free regions of the interface.

In this paper we investigate the impact of interface biasing on the coercive field, the exchange bias, and the switching mechanisms of iron dots. We restrict the analysis to dots with square $\left(21 \times 21 \mathrm{~nm}^{2}\right)$ interface base, and height $(h)$ of the order of the bulk iron exchange length. The point of choosing dots with small interface area is that one may investigate the impact of the dots height and the strength of the interface field on the hysteresis loop shape and the nature of the switching mechanisms.
The effects of geometrical confinement on the magnetic states of soft magnetic materials are controlled by the dipolar and exchange fields. ${ }^{26,27}$ Interface biased flat dots may have new magnetic states if the interface exchange energy is comparable to the intrinsic exchange energy. Also, if the dot's height is larger than the exchange length, the dot's magnetic phases may be affected by the exchange coupling with the substrate spins even though the interface exchange energy is small. This length scale is also interesting from the applications viewpoint. For iron the value of the exchange length is $11.2 \mathrm{~nm},{ }^{28}$ and storage densities in the Tbit in ${ }^{2}$ range require dot arrays with a periodicity of $25 \mathrm{~nm}$.

We show in this paper that unbiased $h=12 \mathrm{~nm}$ and $h$ $=15 \mathrm{~nm}$ iron dots have larger coercivities than the $h$ $=18 \mathrm{~nm}$ dot, and that relevant changes in the hysteresis loops of $h=18 \mathrm{~nm}$ iron dots require values of the interface field strength much smaller than what is required for $h$ $=12 \mathrm{~nm}$ dots. We also show that the differences between flat dots, with heights of $h=12 \mathrm{~nm}$ and $h=15 \mathrm{~nm}$, and $h$ $=18 \mathrm{~nm}$ dots, originate in the nature of the magnetic phases produced by the dipolar field. The adjustment of the magnetization profile to the exchange and dipolar field of unbiased flat dots, with height of the order of the exchange length, result in buckle states [see Fig. 1(a)], while the magnetization patterns of $h=18 \mathrm{~nm}$ unbiased dots, with almost two exchange length in height, are twisted states [see Fig. 1(c)] which are more easily modified by the interface field. We show that major changes in the loops of flat dots require interface field strength of the order of $26 \%$ of the intrinsic exchange field, while for $h=18 \mathrm{~nm}$ dots, noticeable interface features require interface field strength 1 order of magnitude smaller.

\section{THEORETICAL MODEL}

We consider a single Fe dot with a square base of edge $\ell=21 \mathrm{~nm}$, in the $x y$ plane, and height $h$ in the $12-18 \mathrm{~nm}$ range. We chose the dots uniaxial anisotropy easy axis parallel to the interface field along the in-plane $x$ axis, and assume that the antiferromagnetic substrate is held frozen in 


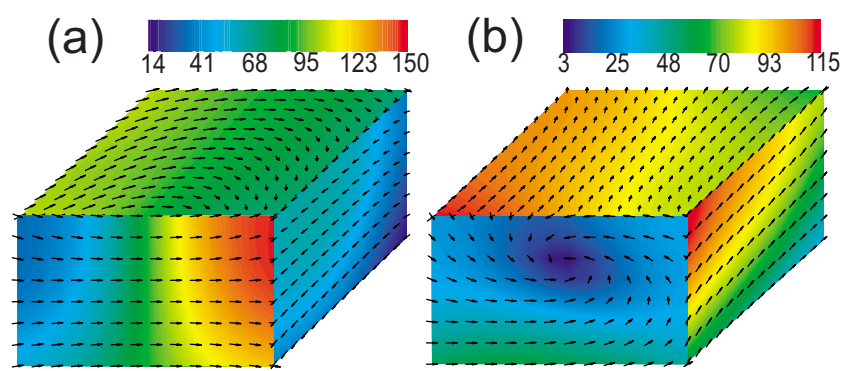

(c) $\begin{array}{lllllll}10 & 36 & 62 & 88 & 114 & 140\end{array}$

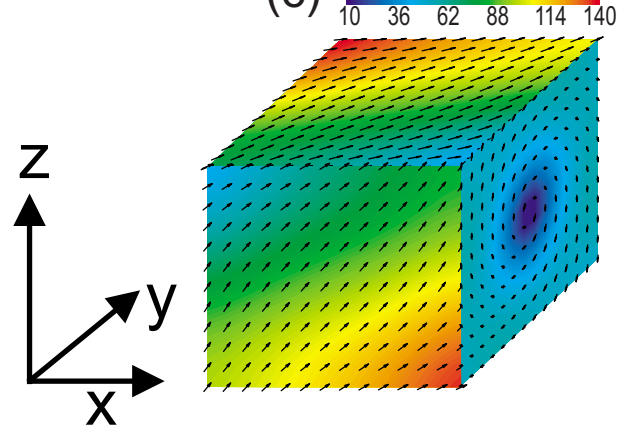

FIG. 1. (Color online) Magnetic states of interface biased iron dots, showing (a) buckle state of $h=12 \mathrm{~nm}$ iron dot for small values of the interface field strength, (b) interface induced twisted state of $h=12 \mathrm{~nm}$ iron dot for large values of the interface field strength, and (c) twisted state of $h=18 \mathrm{~nm}$ iron dot induced by the dipolar field. The color bar code indicates the angle with the normal of each face.

the antiferromagnetic order. The interface exchange coupling of the dot's spins with the AF substrate is represented by effective field acting only on the dot's interface spins. This is a good approximation for large anisotropy antiferromagnets such as $\mathrm{FeF}_{2} \cdot{ }^{16-19}$ It is also valid for other AF substrates. $\mathrm{NiO}$, for instance, is frequently used for biasing purposes, and, even at high temperatures, the relaxation effects require a few hours to produced appreciable changes in the effective exchange bias. $^{20}$

The magnetic structure is described in terms of the magnetic moments of small cubic cells with edge, $d$, smaller than the iron exchange length. The energy density is written as a sum of intrinsic exchange energy, interface exchange energy, anisotropy, Zeeman and dipolar energies:

$$
\begin{aligned}
E= & \frac{A}{d^{2}} \sum_{j} \sum_{k}\left(1-\hat{m}_{j} \cdot \hat{m}_{k}\right) \\
& -M_{S} \vec{H}_{I} \cdot \sum_{i} \hat{m}_{i}-M_{S} \vec{H} \cdot \sum_{j} \hat{m}_{j}-K \sum_{j}\left(\hat{m}_{j}^{x}\right)^{2} \\
& +\frac{M_{S}^{2}}{2} \sum_{j} \sum_{k}\left[\frac{\hat{m}_{j} \cdot \hat{m}_{k}}{n_{j k}^{3}}-\frac{3\left(\hat{m}_{j} \cdot \hat{n}_{j k}\right)\left(\hat{m}_{k} \cdot \hat{n}_{j k}\right)}{n_{j k}^{5}}\right],
\end{aligned}
$$

where the first term is the intrinsic exchange energy, restricted to nearest-neighbor cells, $A$ is the ferromagnetic exchange stiffness, and the effective exchange strength scales as $d^{2}$. The second term is the interface exchange energy, and the sum is restricted to the first layer of cells, corresponding to the interface region. The third term is the Zeeman energy, the fourth term is the anisotropy energy and the last term is the magnetostatic energy. $M_{S}$ is the saturation magnetization, $\hat{m}_{i}$ is the direction of the magnetic moment of the $i$ th cell, and $n_{i j}$, in the last term, is the distance between the cells $i$ and $j$ in units of cell size $(d)$. We use a simulation cell with edge $d=1.5 \mathrm{~nm}$ and the following values for the iron parameters: exchange stiffness $A=1.25 \times 10^{-11} \mathrm{~J} / \mathrm{m}$, anisotropy constant $K=4.7 \times 10^{4} \mathrm{~J} / \mathrm{m}^{3}$ and saturation magnetization $M_{S}=1.7 \times 10^{6} \mathrm{~A} / \mathrm{m}^{29,30} \mathrm{We}$ comment on the value for $A$. Typical values for bulk Fe range from $1.8 \times 10^{-11}$ to 2.5 $\times 10^{-11} \mathrm{~J} / \mathrm{m} .{ }^{31}$ However there is a report that $A$ is about half its bulk value in thin Fe films with thicknesses of $14 \mathrm{~nm}$ (Ref. 32) and we have used this in our choice for $A$.

Size effects are incorporated in the model since the magnetic moments in surface cells have the exchange energy reduced by the absence of nearest neighbors. The equilibrium states are found using a local-field algorithm, which allows calculating the orientation of the magnetic moment in each cell in a self-consistent manner. ${ }^{33-40}$ For each value of the external field strength, the equilibrium configuration is found by seeking a set of directions of the moments in all cells $\left(\hat{m}_{i}, i=1, \ldots, N\right)$ which makes the torque in each one of them equal zero, within a reasonable numeric precision. This amounts to finding values of $\hat{m}_{i}(i=1, \ldots, N)$ for which the magnetic moment of each cell is parallel to the cell local field. The effective local field acting in the $i$ th cell is obtained from the energy density in the usual way: $\vec{H}_{\text {eff }}^{i}$ $=-\left(1 / M_{S}\right)\left(\partial E / \partial \vec{m}_{i}\right)$. We start the calculation at large magnetic fields, where a nearly saturated state is expected. For each value of the external field, the self-consistent procedure is initialized with the magnetic state corresponding to the equilibrium state of the previous value of the external field. Proceeding this way we find the metastable equilibrium state nearest to the preceding one, as appropriate to modeling hysteresis phenomena. Convergence is checked to guarantee a maximum torque of $10^{-26} \mathrm{~J}$ in any one of the cells.

\section{RESULTS}

Before entering the detailed discussion of interface biasing effects in the hysteresis loops, it is instructive to describe the general features of the magnetic states. The typical magnetic states are shown in Fig. 1. In the absence of interface exchange interaction the hysteresis loop shape, the coercivity, and the switching mechanisms are controlled by the dot's dipolar field. The reduction in exchange field near the surfaces make surface moments softer and more easily turned by the dipolar field, leading to a reduction in the component of the magnetization in the direction perpendicular to the surface.

Geometrical confinement in flat dots produces a small curling of the magnetization within each layer to minimize the magnetic charge at the dots surfaces, leading to a buckle configuration, as shown in Fig. 1(a), with spins in the layers parallel to the interface and no appreciable difference between the magnetic structure of the surface layers and the inner layers. Flat dots in the buckle state have an average dipolar field larger than the anisotropy field. Furthermore, the dipolar field is in the direction of the magnetization every- 

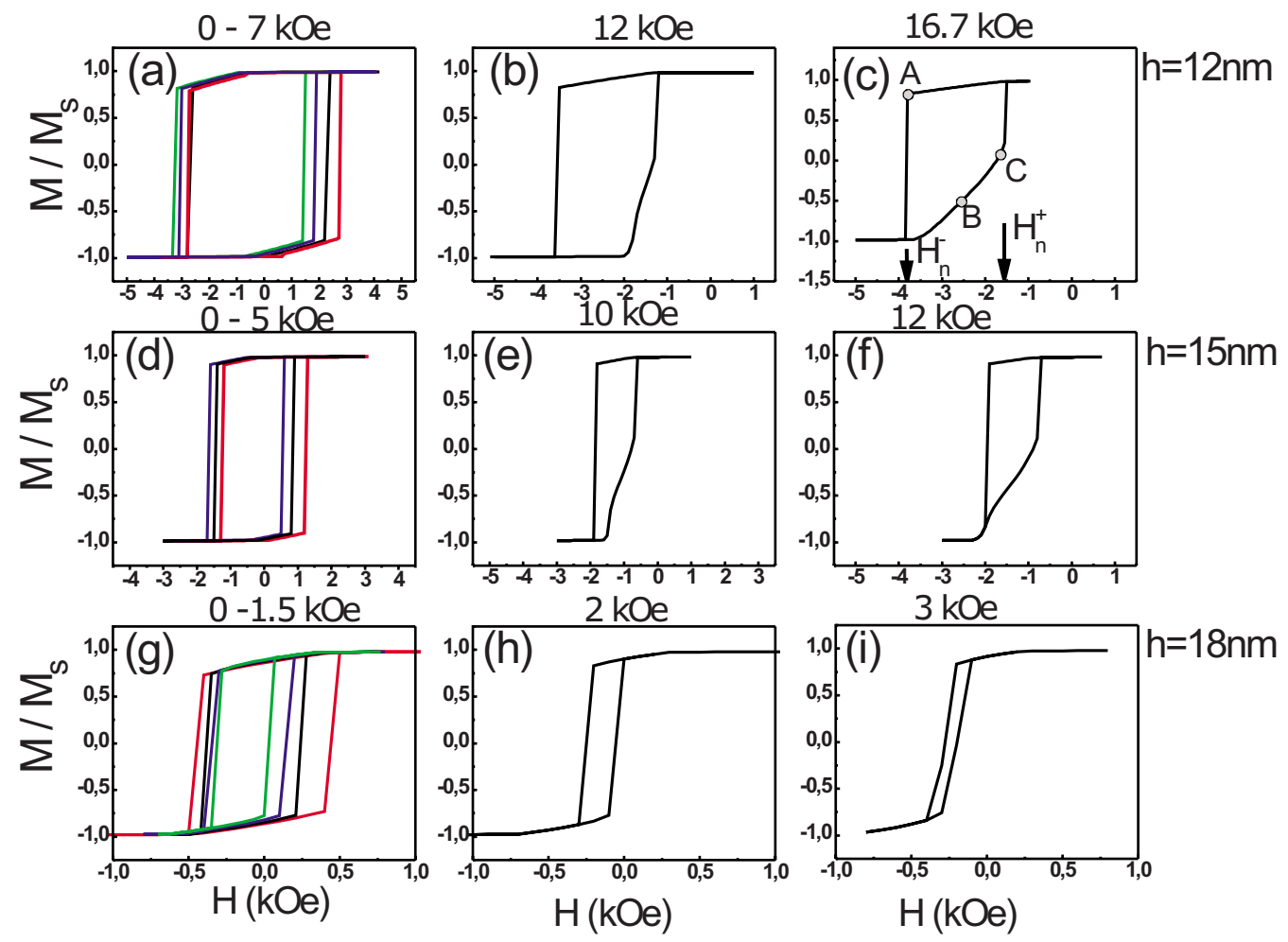

FIG. 2. (Color online) Hysteresis loops for Fe dots with dimensions $21 \times 21 \times h \mathrm{~nm}^{3}$. The numbers on the right of each row of panels, and on the top of columns, indicate the values $h$ and of the interface field strength. For $h=12 \mathrm{~nm}$ the hysteresis shifts rigidly for interface field in the interval $0-7 \mathrm{kOe}$ and for $h=15 \mathrm{~nm}$ the hysteresis shifts rigidly for interface field in the interval $0-5 \mathrm{kOe}$. For $h=18 \mathrm{~nm}$, there is a large reduction in coercivity for an interface field strength of $2 \mathrm{kOe}$.

where but near the charged surfaces, leading to symmetrical Stonner Wolfarth-type hysteresis loops, which may be much wider than those of thin iron films with in-plane anisotropy. Changes in this basic picture require large values of the interface field strength.

Strong interface pinning favors noncoherent switching in flat dots. Noncoherent switching corresponds to the nucleation of twisted states (TS), as shown in Fig. 1(b), in which the average direction of spins in neighboring layers make small angles, and most of the spins in each layer are nearly parallel. For an overall twist of $\left(\theta_{\mathrm{TS}}\right)$ between the interface and free-surface layers there is a cost of exchange energy per unit area, of the order of $\varepsilon(h)=A \theta_{\mathrm{TS}}^{2} / h$, where $A$ is the iron exchange stiffness constant. The nucleation of TS requires values of the interface exchange energy comparable to $\varepsilon(h)$. We show below that the nucleation of twisted states in $h$ $=15 \mathrm{~nm}$ dots requires a smaller value of the interface field strength than for $h=12 \mathrm{~nm}$ dots. The magnetic profile shown in Fig. 1(b) corresponds to $\theta_{\mathrm{TS}}=\pi / 4$ and is formed at the reversal field [see Fig. 2(c)] in the lower branch of the hysteresis loop of a $h=12 \mathrm{~nm}$ dot for an interface field strength of $16.7 \mathrm{kOe}$ (26\% of the iron intrinsic exchange field).

Figure 1(c) shows a twisted state which is formed in unbiased and interface biased $h=18 \mathrm{~nm}$ dots. Since the height is almost equal to two exchange lengths there is room for accommodation of the dipolar field by suitable rotation of the spins of successive $x y$ layers. There is also a similar rotation of spins in neighboring $x z$ layers in order to minimize the magnetic surface charge in the $x y$ and $x z$ faces of the dot.
This phase is the major state of the $h=18 \mathrm{~nm}$ dot and is responsible for a large difference of the interface effects in $h=18 \mathrm{~nm}$ dot in comparison to flat dots $(h=12 \mathrm{~nm}$ and $h$ $=15 \mathrm{~nm}$ dots).

\section{A. Exchange bias and coercivity}

Exchange bias is a common feature of important applications of nanostructured magnetic systems, such as spin valves and magnetic random access memories. Even though it is a subject more than forty years old, ${ }^{41}$ there is not, so far, a comprehensive model to describe the available experimental data. Most of the experimental reports focus on extended systems. One key unsolved feature is that the measured hysteresis shifts are much smaller than what one might expect from arguments based on exchange energy. This is associated to the complex magnetic structure of the interface of extended systems, with lateral dimensions much larger than the exchange length of the ferromagnetic material. Defects, grains, etc. have an impact in the effective interface exchange coupling and switching mechanisms, and have been the basis of the current theoretical models. ${ }^{42,43}$

The interface structure of nanometric dots on an uncompensated AF substrate is likely to be simpler, allowing a better assessment of the impact of the F/AF interface exchange coupling on the hysteresis loops of the ferromagnet. We explore this possibility and investigate the coercive field and exchange bias in a wide interval of values of the interface field strength, starting with unbiased dots and going up to 

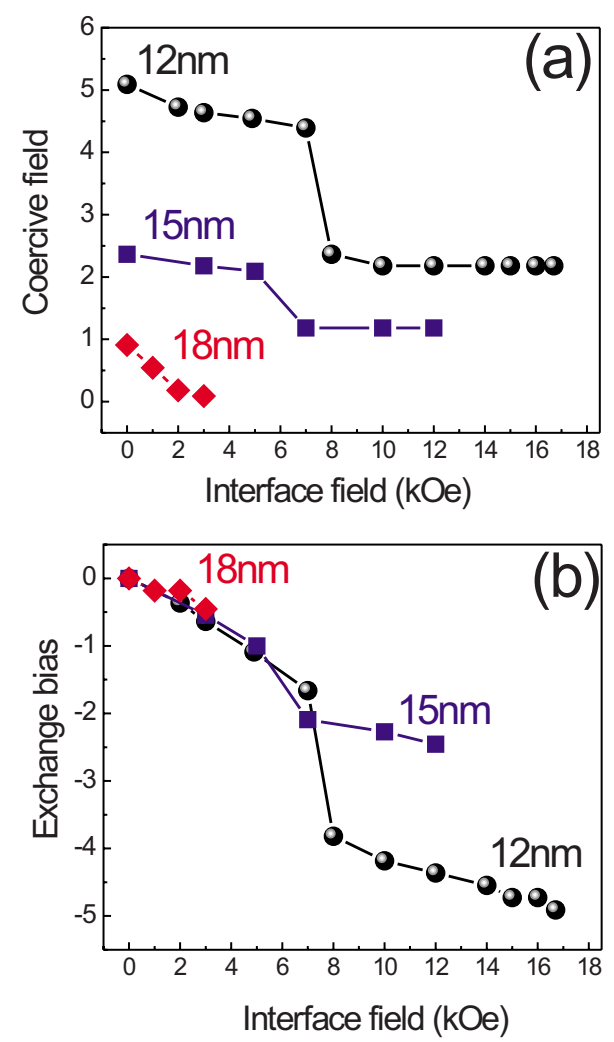

FIG. 3. (Color online) (a) Coercive field and (b) exchange bias shift of flat iron dots. The coercive field $\left(H_{C}\right)$ and the exchange bias field $\left(\mathcal{H}_{\mathrm{EB}}\right)$ are shown in units of the iron uniaxial anisotropy field $\mathcal{H}_{A}$, and the numbers in the figures indicate the dots height $h$. The curves interpolating the calculated points are a guide to the eyes.

values of the interface field strength comparable to that of the intrinsic exchange field of iron (acting on a cell) $\mathcal{H}_{\text {exch }}$ $=2 A / M_{S} d^{2}=65.3 \mathrm{kOe}$.

In Figs. 2 and 3 we show the hysteresis loops of iron dots with height $h=12 \mathrm{~nm}, h=15 \mathrm{~nm}$, and $h=18 \mathrm{~nm}$, and interface field strength from zero (unbiased dot) to $16.7 \mathrm{kOe}$, which is $26 \%$ of the intrinsic exchange field of iron. The data in Fig. 3 were extracted from the values of the external field where major jumps in the magnetization occur, in both branches of the loops, as indicated in Fig. 2(c). Coercivity and bias were obtained from the values of the nucleation fields for reversal in the upper and lower branches $\left(H_{n}^{-}\right.$and $\left.H_{n}^{+}\right)$in the usual manner: $H_{C}=\frac{1}{2}\left(H_{n}^{+}-H_{n}^{-}\right)$, and $H_{C}=\frac{1}{2}\left(H_{n}^{+}\right.$ $\left.+H_{n}^{-}\right)$. The interval of interface field strength in each curve in Fig. 2 is restricted to the maximum value of $\mathcal{H}_{I}$, for which there is a single loop hysteresis and the nucleation fields are clearly defined, as in the loop shown in Fig. 2(c).

Unbiased iron dots with $h=12 \mathrm{~nm}$ have a coercive field of $2.7 \mathrm{kOe}$ which is larger than the iron uniaxial anisotropy field $\left(\mathcal{H}_{A}=550 \mathrm{Oe}\right)$ by a factor of 5 . The coercivity enhancement is a result of shape anisotropy, as we discuss in detail further below. Almost the same coercive field is found if the interface field strength is smaller than $7 \mathrm{kOe}$. There is a large drop in coercivity and a relevant increase in the value of the exchange bias of the $h=12 \mathrm{~nm}$ dot for an interface field strength of approximately $8 \mathrm{kOe}$. The hysteresis loops of the $h=15 \mathrm{~nm}$ iron dot display similar features. In both cases the drop in coercivity occurs at the minimum value of interface field strength required for the nucleation of TS.

For $\mathcal{H}_{I}<7$ kOe $(10.7 \%$ of the intrinsic exchange field) the exchange bias shift $\left(\mathcal{H}_{\mathrm{EB}}\right)$ is proportional to the interface field strength, and is nearly the same for $h=12 \mathrm{~nm}$ and $h$ $=15 \mathrm{~nm}$ dots. The exchange bias shift is approximately equal to $\mathcal{H}_{I} / N$, where $N$ is the number of layers along the normal to the interface.

For small values of the interface field strength, the magnetization reversal of flat dots is a nearly coherent rotation. This is shown in the set of hysteresis loops in Fig. 2(a) for $h=12 \mathrm{~nm}$ iron dots, and in Fig. 2(d) for $h=15 \mathrm{~nm}$ iron dots, for interface strengths below the threshold value for nucleation of twisted states. As shown in Figs. 2(a) and 2(d) the hysteresis loops shift rigidly as the value of $\mathcal{H}_{I}$ is increased, and the exchange bias shift is proportional to the value of $\mathcal{H}_{I}$, as shown in Fig. 3(b).

The formation of twisted states in the up-field branch of the hysteresis loop leads to asymmetrical loops. The threshold values of the interface field strength for nucleation of twisted states may be seen in Fig. 3 from the drop of coercivity and enhancement of the exchange bias shift. For $h$ $=12 \mathrm{~nm}$ dots, the asymmetrical loops are formed if the interface strength is above $8 \mathrm{kOe}$, whereas for $h=15 \mathrm{~nm}$ dots, with a little more room to accommodate the intrinsic exchange energy, the formation of asymmetrical loops requires a smaller value of the interface field strength $(7 \mathrm{kOe}$, or $10.7 \%$ of $\left.\mathcal{H}_{\text {exch }}\right)$. Notice, from Fig. 2, that the asymmetry of the loops requires values of the interface field strength which are of the order of a fraction of the intrinsic exchange field of bulk iron $\left(\mathcal{H}_{\text {exch }}=65.3 \mathrm{kOe}\right)$.

Interface pinning produce much stronger effects in the hysteresis loop of the $h=18 \mathrm{~nm}$ dot. The height of the dot is almost twice the value of the iron exchange length, allowing the formation of twisted states in the unbiased dot. The hysteresis loops shrink when the interface field strength increases. The coercive field of the $h=18 \mathrm{~nm}$ unbiased dot is $0.5 \mathrm{kOe}$ and for $\mathcal{H}_{I}=2 \mathrm{kOe}\left(3 \%\right.$ of $\left.\mathcal{H}_{\text {exch }}\right)$ the coercive field drops to $0.1 \mathrm{kOe}$. We note that these results are consistent with the data reported in early studies of nanostructuring of nanoporous $\mathrm{FeF}_{2} / \mathrm{Fe}$ bilayers. ${ }^{19}$

\section{B. Switching}

In Fig. 4 we show typical magnetic states of an $h$ $=12 \mathrm{~nm}$ dot. Each row of panels in Fig. 4 corresponds to a point in the loop of Fig. 2(c). The buckle state shown in top row of panels in Fig. 4, corresponding to the switching point of the upper branch of the hysteresis loop in Fig. 2(c), is nearly uniform, with a magnetization of $M \approx 75 \% M_{S}$. All layers but the surface layer have the spins in the plane of the layers. Figures 4(b) and 4(c) show the magnetic structure of the twisted states that form in the lower branch of the loop. Notice that the angle of twist $\left(\theta_{\mathrm{TS}}\right)$ decreases as the external field increases in the lower branch of the loop, with the interface layer spins rotating faster to align with the external field than the surface spins. In the lower branch of the loop spin reversal toward the external field direction is nucleated at the interface region. Interface spins start switching first, 
(a)

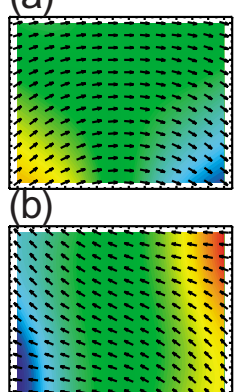

(c)

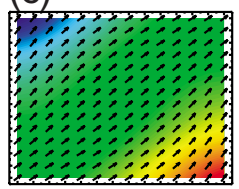

interface
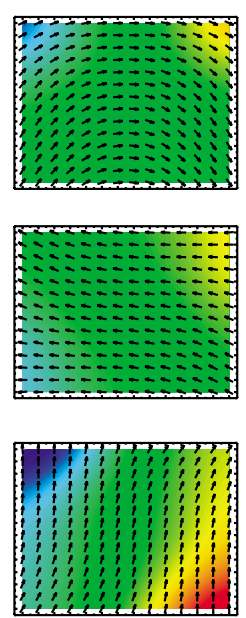

middle
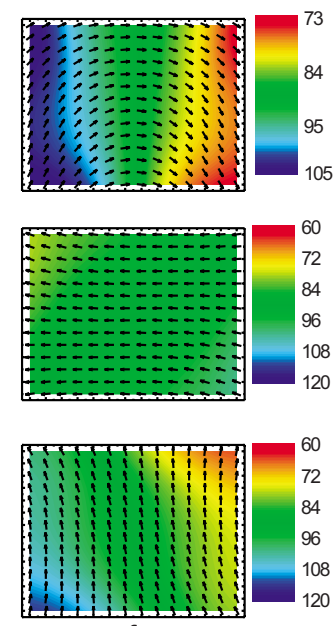

surface

FIG. 4. (Color online) Maps of spin configurations at the interface layer, middle layer, and surface layer, for selected points of the hysteresis loop of Fig. 2(c), for an iron dot with dimensions 21 $\times 21 \times 12 \mathrm{~nm}^{3}$ and $\mathcal{H}_{I}=16.7 \mathrm{kOe}$. The color bar code, in each row of panels, represent the angle of the spins in each layer with the $z$ axis.

dragging the remaining spins via the intrinsic exchange coupling.

The reversal in the upper branch of the loops of flat dots is almost entirely controlled by the dipolar field. As seen in Fig. 2 the field for reversal of the $h=12 \mathrm{~nm}$ varies from $H_{n}^{-}$ $\approx-2.72 \mathrm{kOe}$, for unbiased dots, to $H_{n}^{-} \approx-4 \mathrm{kOe}$, for an interface field strength of $H_{I}=16.7 \mathrm{kOe}\left(25 \%\right.$ of $\left.\mathcal{H}_{\text {exch }}\right)$, while for the $h=15 \mathrm{~nm}$ dot it varies from $H_{n}^{-} \approx-1.5 \mathrm{kOe}$, for unbiased dots, to $H_{n}^{-} \approx-2 \mathrm{kOe}$, for an interface field strength of $\mathcal{H}_{I}=12 \mathrm{kOe}\left(18 \%\right.$ of $\left.\mathcal{H}_{\text {exch }}\right)$. In both cases, for any value of the interface field strength, the magnetic state at the switching field is a buckle state. It has a key role in the magnetization reversal at $H=H_{n}^{-}$, and the large values of coercivity for small values of $H_{I}$ [see Figs. 2(a) and 2(d)].

The magnetostatic energy decreases significantly when surface charges are converted into volume charges. ${ }^{10,44-47}$ The interplay between the surface and volume magnetic charges leads to the strong shape anisotropy of small area flat iron dots. The significant enhancement of coercivity of $h$ $=12 \mathrm{~nm}$ dots is due to volume charges located near the surfaces perpendicular to the net magnetization direction, where there is a small curling of magnetization (in the buckle state).

It is instructive to explore the symmetry of the volume magnetic charges to understand the large increase in coercivity. The volume density of magnetic charge at a given cell is obtained from the variations of the components of the magnetization within the shell of first neighbor cells. In all figures below the volume density of magnetic charge is given in units of $M_{S} / d$. For $\mathrm{Fe}$, with a simulation cell of size $d=1.5 \mathrm{~nm}$, this amounts to a scale factor of 1.1 $\times 10^{15} \mathrm{~A} / \mathrm{m}^{2}$.

In order to have a simple view of the role of the dipolar field on coercivity of flat dots, we have selected to start with a discussion of dipolar effects on the magnetic states of an unbiased $h=12 \mathrm{~nm}$ dot. These results will be used further
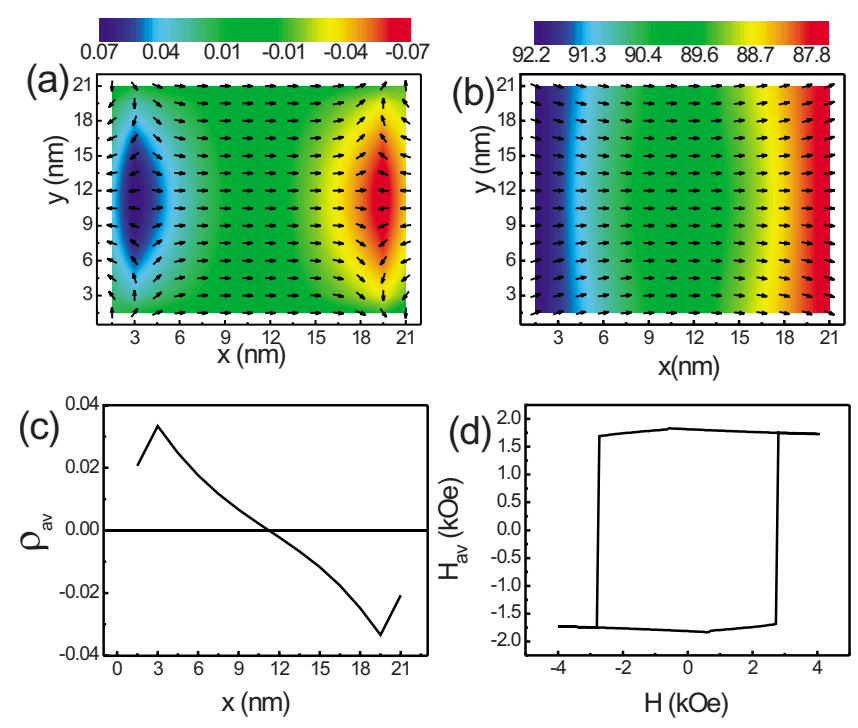

FIG. 5. (Color online) Schematic representation of (a) the dipolar field superimposed to a color map of the volume density of magnetic charge $\rho=-\nabla \cdot \vec{M}$ and (b) the spin configuration, in the middle layer $\left(I_{z}=5\right)$ of an unbiased $h=12 \mathrm{~nm}$ iron dot, with a 21 $\times 21 \mathrm{~nm}^{2}$ base; (c) average volume charge density within cell stripes with fixed value of $I_{x}$; and (d) average dipolar field along the hysteresis loop. The color bar code in panel (a) indicates the value of $\rho\left(I_{x}, I_{y}, I_{z}\right)=-\nabla \cdot \vec{M}$, and in panel (b) indicates the angle of the spins with the $z$ axis.

below as a reference to discuss the effect of interface biasing on the nucleation of reversal.

In Fig. 5 we show the maps of spins and dipolar field at the middle layer of an unbiased $h=12 \mathrm{~nm}$ iron dot, for $H$ $=0$ in the upper branch of the hysteresis loop. The volume charge density, spin, and dipolar field structure shown for the middle layer in Fig. 5 were also found for the other layers, with minor modifications. In Fig. 5(a) we show the map of the volume charge density superimposed to the map of the dipolar field. The corresponding spin map is shown in Fig. 5(b). There is a concentration of positive volume charge near the left $x$ surface (the $y z$ face at $x=0$ ), where the surface charge $(\sigma=\vec{M} \cdot \hat{x}$, not shown in the picture) has a negative value. Also, near the right $x$ surface (the $y z$ face at $x$ $=21 \mathrm{~nm})$, where the surface magnetic charge density is positive $(\vec{M} \cdot \hat{x}>0)$, there is a concentration of negative volume charge. The effective magnetic charge in either side of the dot is dominated by the volume charge density and the dipolar field points in the direction of the magnetization for most of the volume of the dot. Thus, the dipolar field adds stability to the magnetic state and a larger external field, as compared to $H_{A}$, is required to switch the dot.

In order to further discuss the effect of geometrical confinement we make use of two auxiliary quantities. The first quantity is an average value of the volume magnetic charge density $\left[\rho_{\mathrm{av}}(x)\right]$, calculated within a cross section of the dot made of cells within a $y z$ layer, with constant value of $x$ (from $x=0$ to $x=21 \mathrm{~nm}$ ). The use of $\rho_{\mathrm{av}}(x)$ to interpret the origin of the dipolar field is suggested by the symmetry of the dipolar field, as seen in Fig. 5(a). $\rho_{\text {av }}(x)$ has been calcu- 
lated for each point in the hysteresis loop, and in Fig. 5(c) we show $\rho_{\text {av }}(x)$ for $H=0$. The other auxiliary quantity is the average value $\left(\mathcal{H}_{\mathrm{av}}\right)$ of the dipolar field along the direction of the external field. $\mathcal{H}_{\mathrm{av}}$ is an average made over all cells and it is calculated for each point in the hysteresis loop. As shown in Fig. 5(d), $\mathcal{H}_{\mathrm{av}}$ displays a hysteresis loop in phase with the loop corresponding to the magnetization. For a given point in the magnetization hysteresis loop, the average dipolar field is given by

$$
\mathcal{H}_{\mathrm{av}}=\frac{1}{N_{x, y, z}} \sum_{D} H_{D}^{x}(x, y, z),
$$

and the average volume charge is defined as

$$
\rho_{\mathrm{av}}(x)=\frac{1}{N_{y} N_{z}} \sum_{y, z}[-\nabla \cdot \vec{M}(x, y, z)],
$$

where $H_{D}^{x}(x, y, z)=\hat{x} \cdot \vec{H}_{D}(x, y, z), N$ is the number of cells in the dot, $N_{y}$ and $N_{z}$ are the number of cells along the $\hat{y}$ and $\hat{z}$ directions, and

$$
\vec{H}_{D}(x, y, z)=M_{S} \sum_{j}\left[\frac{3\left(\hat{m}_{j} \cdot \hat{n}_{j k}\right) \hat{n}_{j k}}{n_{j k}^{5}}-\frac{\hat{m}_{j}}{n_{j k}^{3}}\right]
$$

is the dipolar field at the $k$ cell located at $(x, y, z)$.

As shown in Fig. 5(c), there is an average polarization due to the volume charge density, which shields the dipolar field produced by the surface charges. The net effect is that in most of the volume of the flat dot, the dipolar field is parallel to the magnetic moment. We have found similar profiles of $\rho_{\text {av }}\left(I_{x}\right)$ for $H \neq 0$. Notice that the average dipolar field along the loop is of the order of $2 \mathrm{kOe}$, thus larger than the anisotropy field by a factor of almost four. As shown in Fig. 5(d), the average dipolar field at the switching point in the upper branch of the loop (corresponding to $H=-2.72 \mathrm{kOe}$ ) is $\mathcal{H}_{\mathrm{av}} \approx 1.8 \mathrm{kOe}$. This leads to the large value of the coercive field seen in Fig. 3(a) for $\mathcal{H}_{I}=0$.

In Figs. 6-8, we show the spin and the dipolar field profiles at the switching field for a flat $(h=12 \mathrm{~nm})$ iron dot. The nature of the switching is more easily visualized looking at the dipolar field pattern. One noticeable feature is the formation of a vortex structure in the dipolar field lines. This originates in the volume density of magnetic charge associated to small variations in the equilibrium spin pattern of the buckle state and may, thus, serve as guide to identify the nucleation of reversal. In Fig. 6, we show the profiles for the middle layer of an unbiased dot at the reversal field in the upper branch of the hysteresis loop $\left(H_{n}^{-} \approx-2.72 \mathrm{kOe}\right)$. Similar profiles were found for the other layers. The intensity of the dipolar field varies from 1.3 to $7.8 \mathrm{kOe}$. The parallel orientation of spins and dipolar field, in the region of largest dipolar field intensity, is the cause of the large coercivity. A good fraction of the spins, in the region of magnetization curling, is perpendicular to the dipolar field, and there is a small fraction of the spins opposite to the dipolar field. The magnetic state at the switching field on the lower branch of the hysteresis loop $\left(H_{n}^{-} \approx 2.72 \mathrm{kOe}\right)$ is equivalent to this.

Similar features are seen in Figs. 7 and 8, except for the fact that the layers (from the interface to the surface) are no (a)

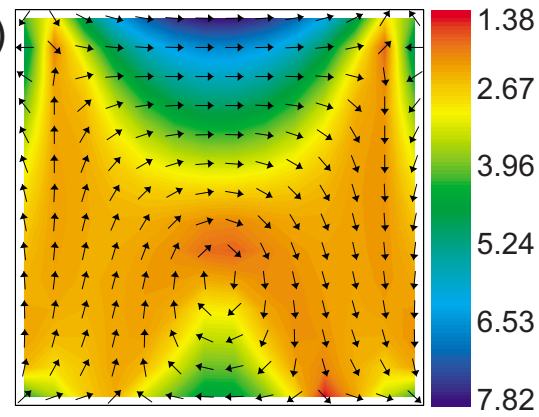

(b)

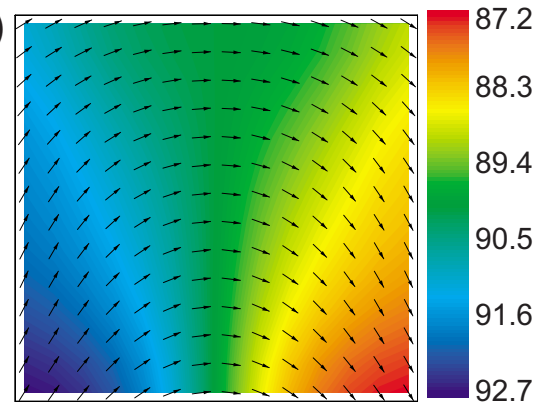

FIG. 6. (Color online) Schematic representation of (a) the dipolar field and (b) the spin configuration of an unbiased $h=12 \mathrm{~nm}$ iron dot, with a $21 \times 21 \mathrm{~nm}^{2}$ base, at the reversal field $\left(H_{n}^{-}\right.$ $=-2.72 \mathrm{kOe}$ ) on the upper branch of the loop. The color bar code indicates (a) the intensity of the dipolar field and (b) the angle of the spins with the $z$ axis.

longer equivalent. Interface biasing introduces asymmetry in the switching modes. The spin reversal starts near the free surface of the dot in the upper branch of the loop and at the interface region in the lower branch of the loop.

In Fig. 7 we show the magnetic structure of a flat $(h$ $=12 \mathrm{~nm}$ ) iron dot for an interface field strength of $\mathcal{H}_{I}$ $=7 \mathrm{kOe}\left(10.7 \%\right.$ of $\left.\mathcal{H}_{\text {exch }}\right)$, at the reversal field, $H_{n}^{-}$ $=-3.17 \mathrm{kOe}$, on the upper branch of the hysteresis loop. The

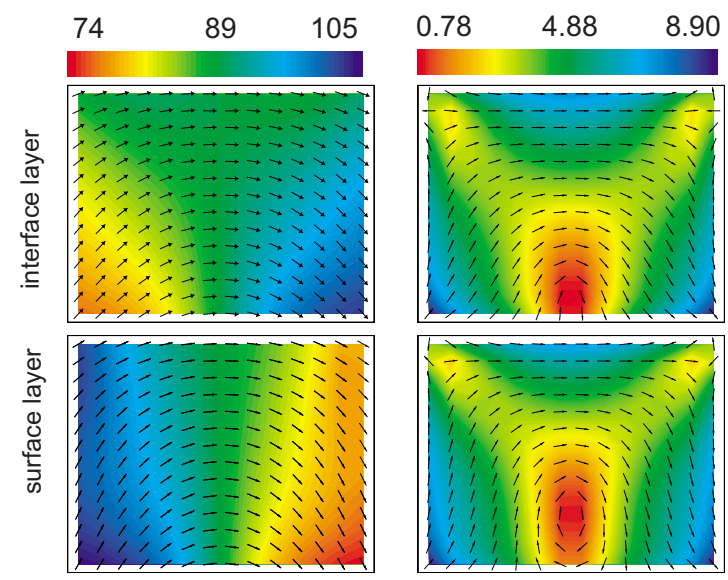

FIG. 7. (Color online) Schematic representation of the spin configuration (panels on the left column) and dipolar field (panels on the right column) of an $h=12 \mathrm{~nm}$ iron dot, with a $21 \times 21 \mathrm{~nm}^{2}$ base, and $\mathcal{H}_{I}=7 \mathrm{kOe}$ at the reversal field $\left(H_{n}^{-}=-3.17 \mathrm{kOe}\right)$ on the upper branch of the loop. The color bar code the left column panels indicates the angle with the $z$ axis, and in the right column panels indicates the intensity of the dipolar field. 

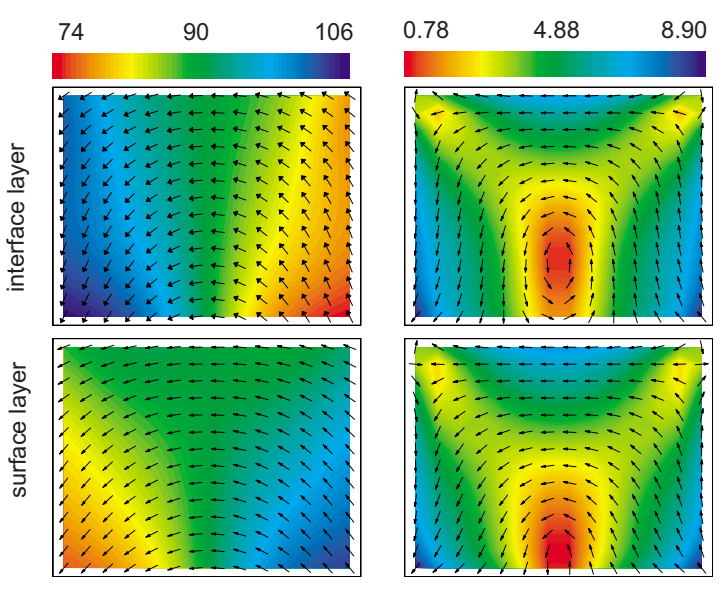

FIG. 8. (Color online) Schematic representation of the spin configuration (panels on the left column) and dipolar field (panels on the right column) of an $h=12 \mathrm{~nm}$ iron dot with $\ell=21 \mathrm{~nm}$ and $\mathcal{H}_{I}=7 \mathrm{kOe}$ at the reversal field $\left(H_{n}^{+}=1.4 \mathrm{kOe}\right)$ on the lower branch of the loop. The color bar code in panels in the left column indicates the angle with the $z$ axis, and in the panels in the right column indicates the intensity of the dipolar field.

majority of spins in the interface layer are in the plane of the layer and nearly parallel to the dipolar field. The vortex of the dipolar field lines is restricted to the surface layer, with the intensity of the dipolar field varying from $0.78 \mathrm{kOe}$ in the center of the vortex to $8.9 \mathrm{kOe}$ near the surfaces. In the surface layer, where the nucleation of reversal starts, a large fraction of the spins, in the region of magnetization curling, has an out-of-layer component. Also, the average dipolar torque in the surface layer is larger, with the spins in the regions of large dipolar field making almost right angles with the dipolar field. The roles of the surface and interface layers are interchanged in the reversal at the lower branch of the loop (at $H_{n}^{+}=1.47 \mathrm{kOe}$ ). As seen in Fig. 8, most of the interface spins have an out-of-layer component. Furthermore, the dipolar field torque is larger at the interface layer. Most of the surface layer spins are parallel to the local dipolar field.

The reduction in coercivity and bias for larger values of the interface field strength is due to the formation of twisted states in the lower branch of the loops. Notice from Figs. 2(a)-2(c) that the major effect of increasing the value of $\mathcal{H}_{I}$ is to change the value of the nucleation field for reversal at the lower branch of the loops $\left(H_{n}^{+}\right)$. The value of the nucleation field for reversal in the upper branch of the hysteresis loop depends weakly on the strength of the interface field. As seen in Figs. 2(a)-2(c) a change by a factor larger than two in the strength of the interface field, from $\mathcal{H}_{I}=7$ kOe to $\mathcal{H}_{I}=16.7 \mathrm{kOe}\left(10.7 \%\right.$ to $25 \%$ of $\left.\mathcal{H}_{\text {exch }}\right)$ leads to a small change in the value of $H_{n}^{-}$. The nucleation of the twisted state has a direct effect on coercivity, by reducing the value of $H_{n}^{+}$, and an indirect effect on the exchange bias shift because large changes in $H_{n}^{+}$affect the position of the center of the loop. Large values of $H_{I}$ also enhance the asymmetry of the reversal mechanisms.

The asymmetry in the switching modes is enhanced for large values of the interface field strength. In Figs. 9 and 10 we show the magnetic structure of a flat iron dot $(h$ $=12 \mathrm{~nm}$ ), at the switching fields, for the case where the re-

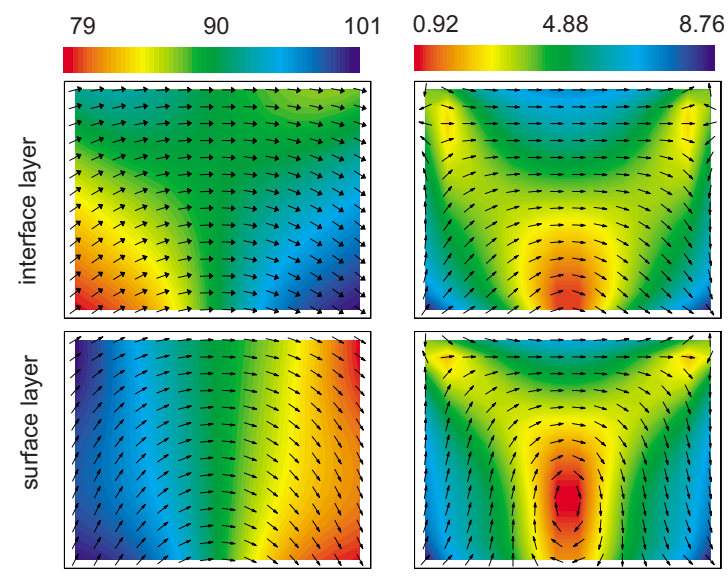

FIG. 9. (Color online) Schematic representation of the spin configuration (panels on the left column) and dipolar field (panels on the right column) of an $h=12 \mathrm{~nm}$ iron dot for an interface field strength of $\mathcal{H}_{I}=16.7 \mathrm{kOe}$ at the reversal field $\left(H_{n}^{-}=-3.8 \mathrm{kOe}\right)$ on the upper branch of the loop. The color bar code in panels in the left column indicates the angle with the $z$ axis, and in the panels in the right column indicates the intensity of the dipolar field.

versal at the lower branch of the hysteresis is mediated by the formation of twisted states, as in the loop shown in Fig. 2(c) for $H_{I}=16.7 \mathrm{kOe}$. As shown in Fig. 9, the reversal at upper branch $\left(H_{n}^{-}=-3.8 \mathrm{kOe}\right)$ is nucleated at the surface layer. The dot is in a buckle state, with a larger curling of the magnetization in the surface layer than in the interface layer. The spins in the surface layer have larger out of layer component and larger dipolar torque.

The reversal at the lower branch of the hysteresis, at $H_{n}^{+}$ $=-1.55 \mathrm{kOe}$ is nucleated at the interface layer. As shown in Fig. 10 the magnetic state at the switching field has a different nature. The interface layer spins switch first, dragging the surface layer spins toward the interface field direction. Also, in this phase the volume charge density is smaller than in the

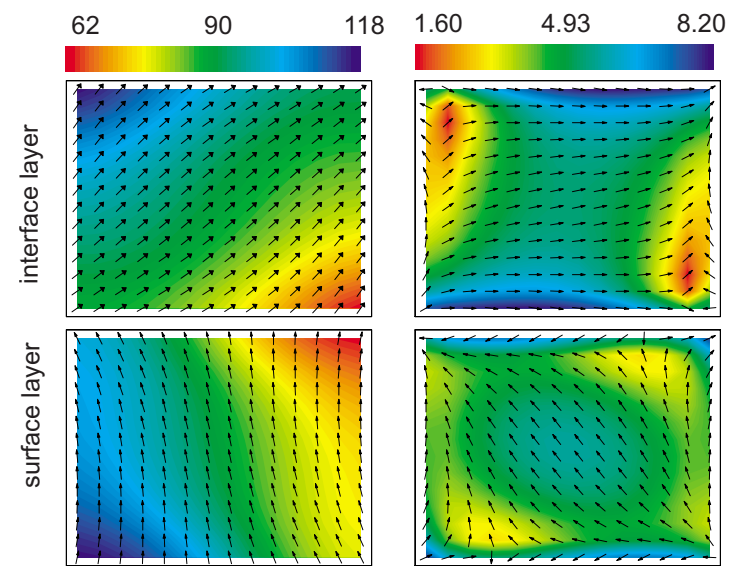

FIG. 10. (Color online) Schematic representation of the spin configuration (panels on the left column) and dipolar field (panels on the right column) of an $h=12 \mathrm{~nm}$ iron dot for an interface field strength of $\mathcal{H}_{I}=16.7 \mathrm{kOe}$ at the reversal field $\left(H_{n}^{+}=-1.55 \mathrm{kOe}\right)$ on the lower branch of the loop. The color bar code in panels in the left column indicates the angle with the $z$ axis, and in the panels in the right column indicates the intensity of the dipolar field. 


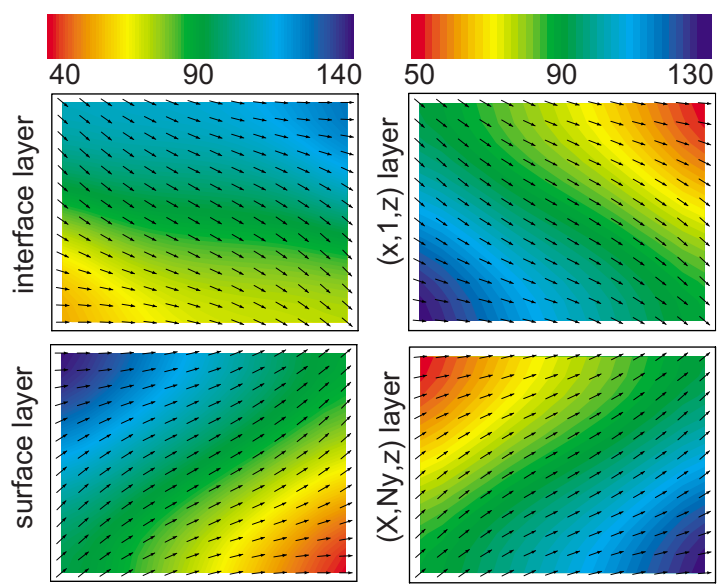

FIG. 11. (Color online) Spin configurations in the $x y$ layers (panels on the left column) and in the $x z$ layers (panels on the right column) of an $h=18 \mathrm{~nm}$ unbiased iron dot with a $21 \times 21 \mathrm{~nm}^{2}$ base for $H=0$. Notice the simultaneous twist of spins in the layers along the $z$-axis direction and $y$-axis direction [see also Fig. 1(c)]. The color bar codes indicate the angle with the normal to the layers $(z$ axis for the panels on the left column, and $y$ axis for the panels on the right column)

buckle state. There is no vortex in the dipolar field. The continuous rotation of the spins from the interface layer to the surface layer leads to a corresponding pattern of the dipolar field.

The shape of the hysteresis loops of $h=18 \mathrm{~nm}$ dots is to a large extent controlled by the dipolar field. Due to the larger height, there is room to relax the magnetic structure along the direction perpendicular to the surface, with a small misalignment of spins in neighboring layers. On the left side of Fig. 11 we show the magnetic structure of the surface and interface layers of an unbiased $h=18 \mathrm{~nm}$ dot for $H=0$. The layer by layer rotation of the average spin direction along the $z$-axis direction occurs with a simultaneous rotation of the average spin direction in the layers along the $y$-axis direction. There is a large out-of-layer component, in both directions, except for spins in the central part of the $x y$ and $x z$. layers, allowing the simultaneous twist of the average spin direction. This magnetic phase is easily modified by the interface field.

In Fig. 12 we show schematically the magnetic structure of all layers, from the interface to the surface, making use of the average spin direction in each $x y$ layer, and the average direction and intensity of the dipolar field in each layer as well. The panel [Fig. 12(b)] corresponding to $H=0$ indicates that the structure is symmetric and that the intensity of the dipolar field is larger at the interface and surface layers $\left(\left\langle H_{d}\right\rangle=4.13 \mathrm{kOe}\right)$, with a minimum at the central layers $\left(\left\langle H_{d}\right\rangle=0.6 \mathrm{kOe}\right)$. The other panels for selected points along the upper branch of the hysteresis loop show that the twisted state is also symmetrical in other points in the loop.

Panel (a) in Fig. 12 shows the magnetic structure at the value of $H$ for which the twisted state is formed. The end spins make angles of $\pm 16^{\circ}$ with the uniaxial axis. This pattern evolves from this rather weakly twisted state, passing through $\pm 27^{\circ}$, for $H=0$, as shown in panel (b), going up to $\pm 44^{\circ}$ for the state where the nucleation of reversal occurs at

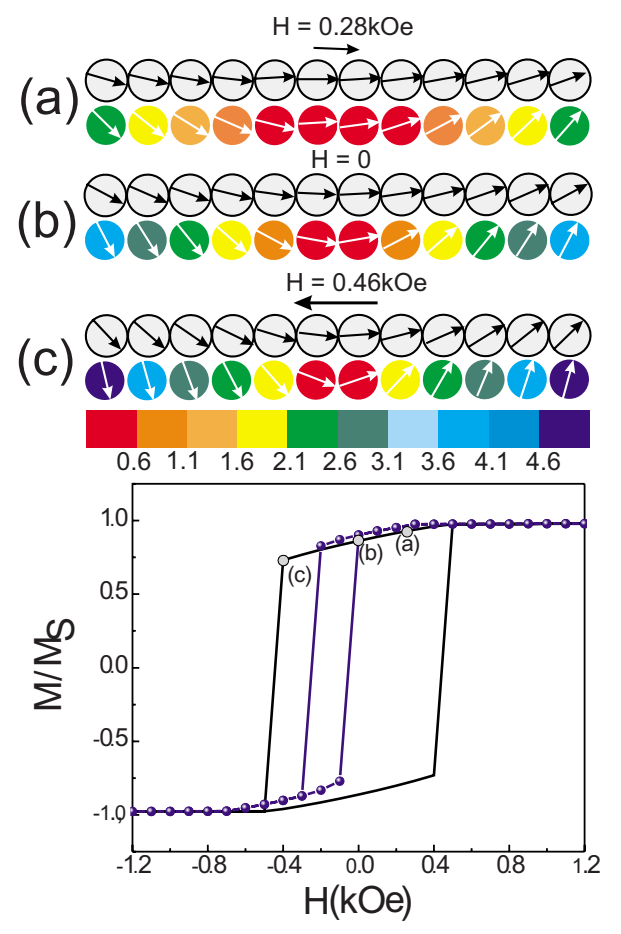

FIG. 12. (Color online) Schematic representation of the magnetic states and dipolar field of an unbiased iron dot with $h$ $=18 \mathrm{~nm}$ for selected points along the hysteresis loop. The panels display the average direction of spins $(\langle S\rangle)$ and dipolar field $\left(\left\langle H_{d}\right\rangle\right)$ in the $x y$ layers, from the interface to the free surface of the dot. The color bar code indicates the intensity of the dipolar field (kOe). The inner hysteresis loop corresponds to an interface field strength of $2 \mathrm{kOe}$.

$H=-0.46 \mathrm{kOe}$, as shown in panel (c). The average intensity of the dipolar field is not uniform across the layers, and at the reversal field the maximum value of the dipolar field is at the surface and interface layers. Notice also that the coercivity decays by a factor of 5 for a small value of the interface field strength $(2 \mathrm{kOe})$.

In Fig. 13 we show schematically the magnetic pattern at the switching fields for an unbiased $h=18 \mathrm{~nm}$ dot and for the interface biased $\left(\mathcal{H}_{I}=2 \mathrm{kOe}\right)$ dot. As seen in Fig. 13, a small value of the interface field produces considerable changes in the magnetic pattern at the switching fields. Comparing the profiles, in Fig. 13(a), at the reversal in the upper branch of the hysteresis $\left(H_{n}^{-}\right)$, we see that the pinning at the interface shifts the center of the twist toward the interface, leading to a larger value of the twist angle at the surface layer. There is also a change in the along the $y$ axis, as shown in Fig. 13(b). These changes in the magnetic structure of the unbiased dot originated in the interface pinning (even for small value of the pinning field) are possible because all the dimensions of the dot are larger than the iron exchange length.

\section{CONCLUSIONS}

Our results indicate that the combined effects of the dipolar and interface field play an important role in the magnetic 

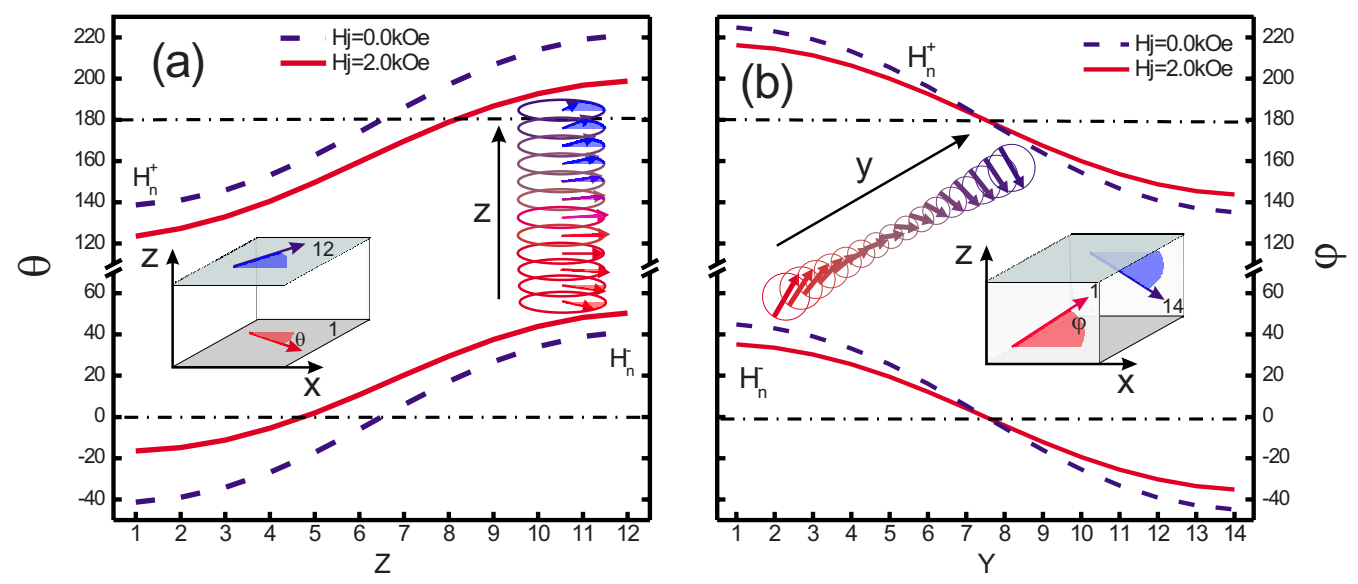

FIG. 13. (Color online) Schematic representation of the twisted state at the reversal fields at the upper $\left(H_{n}^{-}\right)$and lower $\left(H_{n}^{+}\right)$branches of the hysteresis loop of an unbiased (dashed curve) and interface biased (continuous curve) iron dot with $h=18 \mathrm{~nm}$. The angle of the average spin direction within (a) the $x y$ layers and (b) within the $x z$ layers with the uniaxial $x$ axis.

states of dots with a square base and dimensions comparable to the ferromagnetic exchange length. The form of the hysteresis curves may be strongly affected by the dot height, as well as the strength of the interface exchange field.

We have shown that flat dots $(h=12 \mathrm{~nm})$, with height of the order of the iron exchange length, have symmetrical hysteresis curves, even for values of the interface field strength $\left(\mathcal{H}_{I}\right) 1$ order of magnitude larger than the uniaxial anisotropy field. The hysteresis curves turn asymmetrical if the interface field strength is large enough to nucleate twisted states. This is a key feature of the hysteresis of small area dots. The nucleation of twisted states in the lower branch of the loop (external field opposite to the interface field) requires interface field strengths comparable to the intrinsic exchange field of bulk iron. The formation of twisted states may be identified by a large asymmetry of the hysteresis loop, which may, thus, be used as a signature of strong interface exchange energy.

Flat dots have large shape anisotropy. The dipolar field is parallel to the direction of the spins, except for a narrow region near the surfaces. The volume charge density is located near the surfaces and has the opposite polarity of the surface charge. It corresponds to a small curling of the magnetization to minimize the surface charges. The total magnetic charge forms double layers near the charged surfaces, with the volume charge prevailing over the surface charge and leading to the form of the dipolar field inside the dot.
The coercivity and the value of the exchange bias are nonlinear functions of the interface field strength. For small values of the interface field strength the hysteresis shift is nearly the value of the interface field scaled by the dot thickness, which is commonly taken as a guide for theoretical models of exchange bias. However if the interface field strength is large enough to nucleate twisted states, there is a large change in the hysteresis shift.

Relevant changes in the shape of the hysteresis loops of flat dots $(h=12 \mathrm{~nm})$ require large values of the interface field strength. Interface pinning produce much stronger effects in the hysteresis loop of the $h=18 \mathrm{~nm}$ dot. The height of the dot is almost twice the value of the iron exchange length, allowing the formation of twisted states in the unbiased dot. This is a much softer magnetic phase than the buckle phase of flat dots. As a result, even a small value of the interface field strength produces large drops in the coercivity. The hysteresis loops shrink rapidly when the interface field strength increases.

As a final remark, we note that interesting new features, particularly regarding the coercivity and exchange bias, should result by extending the model explored in the present work to dots with various aspect ratios. ${ }^{48}$

\section{ACKNOWLEDGMENTS}

This research was partially supported by the Brazilian research agencies CAPES and CNPq. The work of R.E.C. was supported by DOA Grant No. W911NF-04-1-0247.
${ }^{1}$ B. D. Terris, M. Albrecht, G. Hu, T. Thomson, and C. T. Rettner, IEEE Trans. Magn. 41, 2822 (2005).

${ }^{2}$ J. I. Martín, Y. Jaccard, A. Hoffmann, J. Nogués, J. M. George, J. L. Vicent, and Ivan K. Schuller, J. Appl. Phys. 84, 411 (1998).

${ }^{3}$ M. Hehn, K. Ounadjela, J. P. Bucher, F. Rousseaux, D. Decanini, B. Bartenlian, and C. Chappert, Science 272, 1782 (1996).

${ }^{4}$ J. I. Martín, J. Nogués, Kai Liu, J. L. Vicent, and Ivan K. Schuller, J. Magn. Magn. Mater. 256, 449 (2003).
${ }^{5}$ B. D. Terris, T. Thomson, and G. Hu, Microsyst. Technol. 13, 189 (2006)

${ }^{6}$ C. A. Ross, Annu. Rev. Mater. Res. 31, 203 (2001).

${ }^{7}$ B. D. Terris and T. Thomson, J. Phys. D 38, R199 (2005).

${ }^{8}$ M. Albrecht, G. Hu, A. Moser, O. Hellwig, and B. D. Terris, J. Appl. Phys. 97, 103910 (2005).

${ }^{9}$ J. Vogel, J. Moritz, and O. Fruchart, C. R. Phys. 7, 977 (2006).

${ }^{10}$ R. P. Cowburn, A. O. Adeyeye, and M. E. Welland, Phys. Rev. 
Lett. 81, 5414 (1998).

${ }^{11}$ R. P. Cowburn, D. K. Koltsov, A. O. Adeyeye, M. E. Welland, and D. M. Tricker, Phys. Rev. Lett. 83, 1042 (1999).

${ }^{12}$ T. Shinjo, T. Okuno, R. Hassdorf, K. Shigeto, and T. Ono, Science 289, 930 (2000).

${ }^{13}$ F. Q. Zhu, G. W. Chern, O. Tchernyshyov, X. C. Zhu, J. G. Zhu, and C. L. Chien, Phys. Rev. Lett. 96, 027205 (2006).

${ }^{14}$ H. F. Ding, A. K. Schmid, Dongqi Li, K. Yu. Guslienko, and S. D. Bader, Phys. Rev. Lett. 94, 157202 (2005).

${ }^{15}$ J. Y. Cheng, W. Jung, and C. A. Ross, Phys. Rev. B 70, 064417 (2004).

${ }^{16}$ K. Liu, S. M. Baker, M. Tuominen, T. P. Russell, and Ivan K. Schuller, Phys. Rev. B 63, 060403(R) (2001).

${ }^{17}$ Z. P. Li, O. Petracic, J. Eisenmenger, and Ivan K. Schuller, Appl. Phys. Lett. 86, 072501 (2005).

${ }^{18}$ M. R. Fitzsimmons, P. Yashar, C. Leighton, Ivan K. Schuller, J. Nogués, C. F. Majkrzak, and J. A. Dura, Phys. Rev. Lett. 84, 3986 (2000).

${ }^{19}$ J. Eisenmenger, Zhi-Pan Li, W. A. A. Macedo, and I. K. Schuller, Phys. Rev. Lett. 94, 057203 (2005).

${ }^{20}$ P. A. A. van der Heijden, T. F. M. M. Maas, W. J. M. de Jonge, J. C. S. Kools, F. Roozeboom, and P. J. van der Zaag, Appl. Phys. Lett. 72, 492 (1998).

${ }^{21}$ M. Fraune, U. Rudiger, and G. Guntherodt, Appl. Phys. Lett. 77, 3815 (2000).

${ }^{22}$ E. Girgis, R. D. Portugal, M. J. Van Bael, K. Temst, and C. Van Haesendonck, J. Appl. Phys. 97, 103911 (2005).

${ }^{23}$ L. Sun, Y. Ding, C. L. Chien, and P. C. Searson, Phys. Rev. B 64, 184430 (2001).

${ }^{24}$ Y. Shen, Y. Wu, H. Xie, K. Li, J. Qiu, and Z. Guo, J. Appl. Phys. 91, 8001 (2002).

${ }^{25}$ E. Girgis, R. D. Portugal, H. Loosvelt, M. J. Van Bael, I. Gordon, M. Malfait, K. Temst, C. Van Haesendonck, L. H. A. Leunissen, and R. Jonckheere, Phys. Rev. Lett. 91, 187202 (2003).

${ }^{26}$ A. Hubert and R. Schäafer, Magnetic Domains: The Analysis of Magnetic Microstructures (Springer, Berlin, 1999).

${ }^{27}$ M. E. Schabes and H. N. Bertran, J. Appl. Phys. 64, 1347 (1988).

${ }^{28}$ O. Fruchart, J. P. Nozieres, W. Wernsdorfer, D. Givord, F. Rousseaux, and D. Decanini, Phys. Rev. Lett. 82, 1305 (1999).
${ }^{29}$ F. Porrati and M. Huth, Appl. Phys. Lett. 85, 3157 (2004).

${ }^{30}$ M. Grimsditch, G. K. Leaf, H. G. Kaper, D. A. Karpeev, and R. E. Camley, Phys. Rev. B 69, 174428 (2004).

${ }^{31}$ C. A. F. Vaz, J. A. C. Bland, and G. Lauhoff, Rep. Prog. Phys. 71, 056501 (2008).

${ }^{32}$ R. J. Hicken, D. E. P. Eley, M. Gester, S. J. Gray, C. Daboo, A. J. R. Ives, and J. A. C. Bland, J. Magn. Magn. Mater. 145, 278 (1995).

${ }^{33}$ R. E. Camley, Phys. Rev. B 35, 3608 (1987).

${ }^{34}$ R. E. Camley, J. Kwo, M. Hong, and C. L. Chien, Phys. Rev. Lett. 64, 2703 (1990).

${ }^{35}$ A. S. Carriço and R. E. Camley, Phys. Rev. B 45, 13117 (1992).

${ }^{36}$ A. L. Dantas and A. S. Carriço, Phys. Rev. B 59, 1223 (1999).

${ }^{37}$ M. L. Silva, A. L. Dantas, and A. S. Carriço, Solid State Commun. 135, 769 (2005).

${ }^{38}$ M. L. Silva, A. L. Dantas, and A. S. Carriço, J. Magn. Magn. Mater. 292, 453 (2005).

${ }^{39}$ A. L. Dantas, G. O. G. Rebouças, A. S. W. T. Silva, and A. S. Carriço, J. Appl. Phys. 97, 10K105 (2005).

${ }^{40}$ A. L. Dantas, G. O. G. Rebouças, J. C. P. Barbosa, H. T. Souza, I. S. Queiroz, Jr., and A. S. Carriço, Physica B 353, 287 (2004).

${ }^{41}$ W. H. Meiklejohn and C. P. Bean, Phys. Rev. 105, 904 (1957).

${ }^{42}$ J. Nogués and I. K. Schuller, J. Magn. Magn. Mater. 192, 203 (1999).

${ }^{43}$ J. Nogués, J. Sort, V. Langlais, V. Skumryev, S. Suriñach, J. S. Muñoz, and M. D. Baró, Phys. Rep. 422, 65 (2005).

${ }^{44}$ R. M. Hornreich, J. Appl. Phys. 34, 1071 (1963).

${ }^{45}$ K. Nonaka, S. Hirono, and I. Hatakeyama, J. Appl. Phys. 58, 1610 (1985).

${ }^{46}$ W. Rave, K. Fabian, and A. Hubert, J. Magn. Magn. Mater. 190, 332 (1998).

${ }^{47}$ C. L. Dennis, R. P. Borges, L. D. Buda, U. Ebels, J. F. Gregg, M. Hehn, E. Jouguelet, K. Ounadjela, I. Petej, I. L. Prejbeanu, and M. J. Thornton, J. Phys.: Condens. Matter 14, R1175 (2002).

${ }^{48}$ In a recent study of the thermal hysteresis of interface biased $\mathrm{Fe}$ dots, it was shown that the width of the hysteresis loops depends on the dimensions of the ferromagnetic dot, and can be significant for dots elongated in the direction of the interface field. Ana L. Dantas, A. S. W. T. Silva, G. O. G. Rebouças, A. S. Carriço, and R. E. Camley, J. Appl. Phys. 102, 123907 (2007). 